\title{
Predictions of the Compressible Fluid Model and its Comparison to Experimental Measurements of $Q$ Factors and Flexural Resonance Frequencies for Microcantilevers
}

\author{
Jason Jensen and Martin Hegner \\ Centre for Research on Adaptive Nanostructures and Nanodevices (CRANN) and School of Physics, \\ Trinity College Dublin, Dublin 2, Ireland \\ Correspondence should be addressed to Jason Jensen, jensenja@tcd.ie and Martin Hegner, hegnerm@tcd.ie \\ Received 15 June 2011; Accepted 6 July 2011 \\ Academic Editor: Sangmin Jeon \\ Copyright ( $\odot 2012$ J. Jensen and M. Hegner. This is an open access article distributed under the Creative Commons Attribution \\ License, which permits unrestricted use, distribution, and reproduction in any medium, provided the original work is properly \\ cited. \\ The qualitative agreement between experimental measurements of the $Q$ factors and flexural resonance frequencies in air of \\ microcantilevers and calculations based on the compressible fluid model of Van Eysden and Sader (2009) is presented. The Q \\ factors and resonance frequencies observed on two sets of cantilever arrays were slightly lower than those predicted by the model. \\ This is attributed to the individual design and geometry of the microfabricated hinged end of the cantilever beams in the array.
}

\section{Introduction}

The introduction of the atomic force microscope [1] and the improvement of silicon fabrication technologies resulted in the ready availability of high-quality, reproducible, and inexpensive silicon cantilevers. Applications for micron-scale cantilevers as a sensing tool have been found in the fields of genomics [2-6], proteomics [7-9], microbiology [1014], and many others. Many of these applications make use of the microcantilever as a sensitive mass detector. It has been shown that operating the cantilever at higher resonance modes increases the mass sensitivity of the device [15]. This increase in the sensitivity is linked to the increased $Q$ factor observed for the higher flexural resonance modes of the cantilever [15]. Along with increased interest in possible applications came the need for improved understanding of the dynamics of cantilevers on this scale and models which can predict their behaviour in a range of situations. In general the higher the $Q$ factor of the resonance peak the smaller the minimum observable frequency shift is. Thus it is desirable to obtain the highest $Q$ factor possible during experiments to maximise the sensitivity of the experiment. Models indicating the dynamics of the cantilever are useful when planning such experiments and determining the expected minimum response required for successful detection of the target.

Many models detailing the behaviour of microcantilevers have been proposed, including the Elmer-Dreier model [16] and Sader's viscous [17] and extended viscous models [18]. Sader's extended model includes the 3D flow field of the fluid around the cantilever beam and can be applied for arbitrary mode number.

The models mentioned above assume that the fluid in which the cantilever is vibrating is incompressible, and in general have good agreement with experimental results [19]. However, recent papers by Van Eysden and Sader $[20,21]$ which detail a model for a cantilever beam oscillating in a compressible fluid indicate that this unbounded increase of the quality factor is not always valid. They predict that as the mode number increases and passes a "coincidence point" (which is determined by the thickness to length ratio of the cantilever and the fluid in which the cantilever is vibrating) the $Q$ factor will begin to decrease.

This coincidence point occurs when the length scale of spatial oscillations of the cantilever beam reduces to a point where it is comparable with the acoustic wavelength of the 


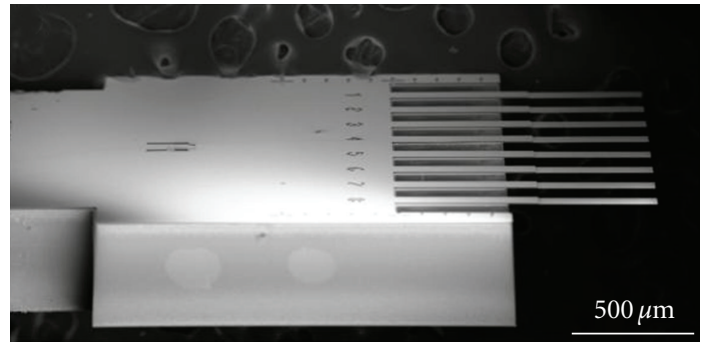

(a)

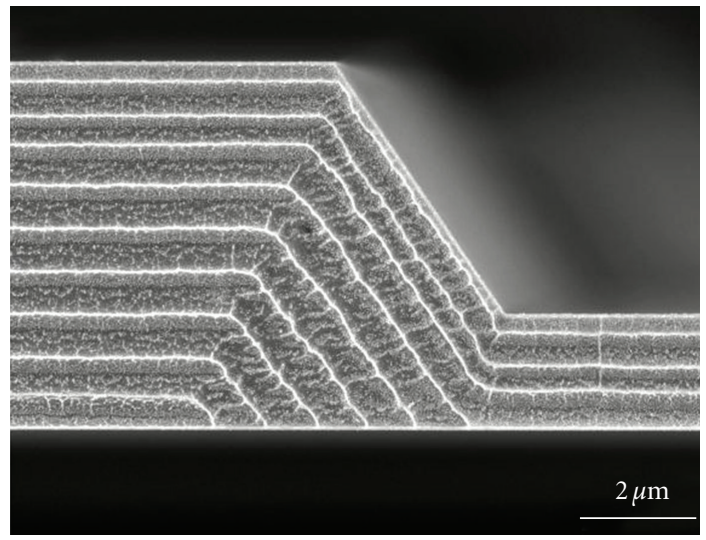

(c)

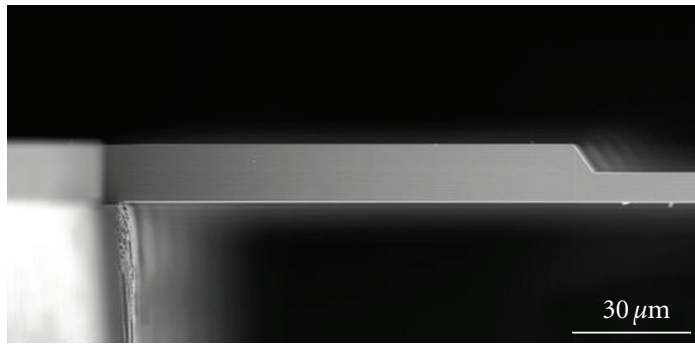

(b)

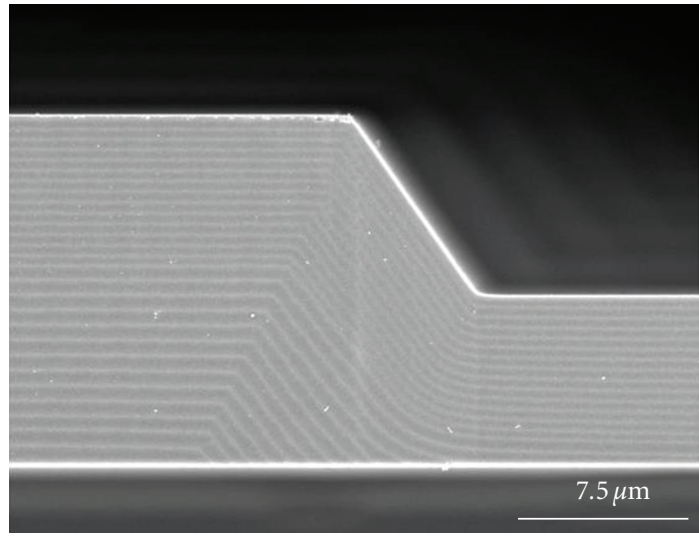

(d)

FIGURE 1: (a) SEM image of typical cantilever array used for these measurements. (b) Closer view of the hinged end of one of the $7 \mu \mathrm{m}$ thick cantilever showing the $120 \mu \mathrm{m}$ long hinged design that connects the cantilever with the main body of the array. (c), (d) Two closer views of the hinged portion of the 2 and $7 \mu \mathrm{m}$ thick cantilevers, respectively. The hinge is approximately twice the thickness of the cantilever for the $7 \mu \mathrm{m}$ thick cantilevers and approximately three times the thickness on the $2 \mu \mathrm{m}$ thick cantilevers.

media in which the cantilever is vibrating. At this point it is possible that energy can be dissipated by the generation of acoustic waves.

For practical applications of microcantilevers (such as mass sensing) this is not an issue when operating the cantilever in liquid. However, if the cantilever is vibrated in air then it can be possible to observe this effect at higher modes. For a cantilever which is $100 \mu \mathrm{m}$ wide, $500 \mu \mathrm{m}$ long, and $7 \mu \mathrm{m}$ thick the scaling analysis from the compressible fluid model [20] predicts that there should be a turning point at the $n=3$ mode which occurs below $1 \mathrm{MHz}$. For a $2 \mu \mathrm{m}$ thick cantilever of the same size the predicted mode is much higher $(n=12)$ and occurs around $3.6 \mathrm{MHz}$.

\section{Materials and Methods}

2.1. Cantilevers. The cantilevers used in these experiments are Si cantilever arrays (orientation: 110) with eight cantilevers per array (IBM Research Laboratory, Rüschlikon, Switzerland). The cantilevers had a pitch of $250 \mu \mathrm{m}$ and were $500 \mu \mathrm{m}$ long and $100 \mu \mathrm{m}$ wide. The thickness of the cantilevers was measured in a scanning electron microscope (SEM, Zeiss Ultra, Cambridge, UK) and were found to be $7.2 \pm 0.5 \mu \mathrm{m}$ and $1.972 \pm 0.005 \mu \mathrm{m}$ thick (Figure 1). The variation in the thicknesses of the cantilevers was shown to depend on their position on the production wafer. As shown in Figure 1 the cantilevers are connected to the main body of the chip via a $\sim 120 \mu \mathrm{m}$ long segment which is approximately twice as thick as the cantilever itself for the $7 \mu \mathrm{m}$ thick cantilevers and three times the thickness for the $2 \mu \mathrm{m}$ thick cantilevers. This design was implemented to facilitate better definition between the hinge (clamping point) of the cantilever and the main body of the array.

2.2. Optical Beam Deflection Device. Thermal actuation of the cantilevers does not provide sufficient vibration of the cantilever beam to allow measurement of higher resonance modes in the current device. The cantilevers are clamped on top of a piezo electric actuator (EBL Products Inc., East Hartford, Conn, USA). The energy from the piezo is efficiently transferred to the cantilevers and provides sufficient vibration amplitudes to allow readout of the vibration modes using optical beam deflection. The cantilevers are excited at various vibrating modes by a linear frequency sweep of a sinusoidal signal which is provided by a frequency generator (NI PCI 5406, National Instruments, Tex, USA) which is controlled via a LabVIEW interface. The drive amplitude of the piezo actuator was kept low to avoid nonlinearities in the response of the cantilevers.

Optical beam deflection was used to detect the resonance frequency of the cantilever vibrations. A schematic of the device is shown in Figure 2. A single wavelength fibre coupled laser $(632.99 \mathrm{~nm}$, Free space power $>2.4 \mathrm{~mW}$, SWL 7504-P; 


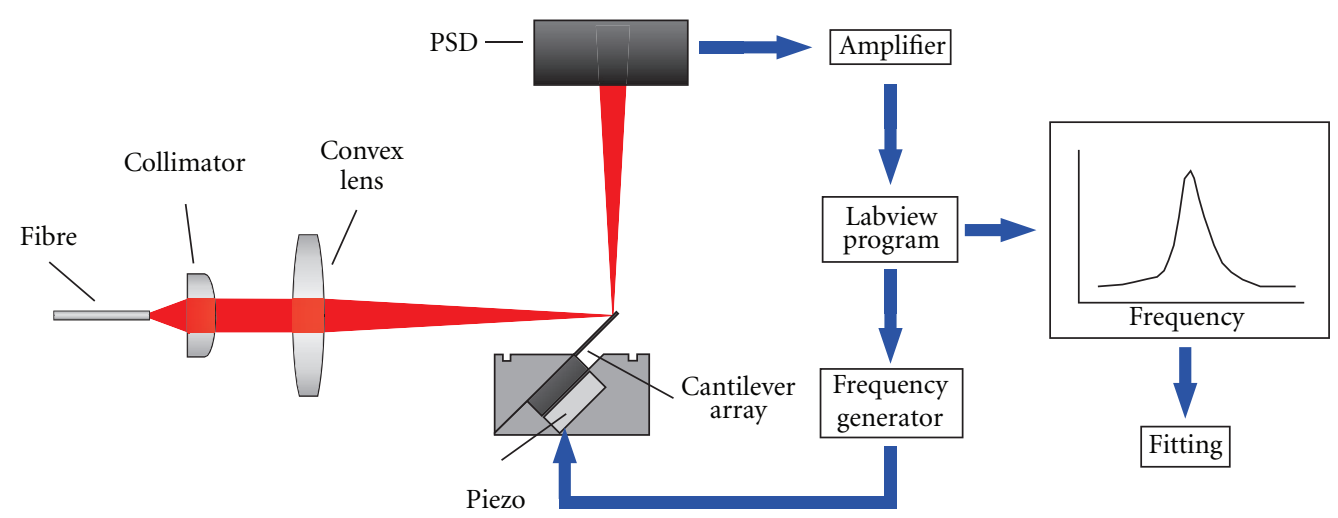

FIGURE 2: Schematic of the optical beam deflection readout procedure. The cantilever array is actuated by a piezo electric ceramic which is excited by a signal from the frequency generator. A laser spot focused onto the tip of the cantilever is deflected onto the surface of a PSD. The output from the PSD is then analysed with the signal from the frequency generator in a LabVIEW program which results in a frequency spectrum, the peaks of which correspond to the flexural resonance modes of the cantilever.

Newport, Calif, USA) was collimated into a $3.5 \mathrm{~mm}$ beam diameter (F280 APC-B; Thorlabs, Cambridgeshire, UK) and then focussed onto a $12 \mu \mathrm{m}$ diameter spot on the surface of the cantilever using a $50 \mathrm{~mm}$ focal length convex lens (AC254-050-A1-ML; Thorlabs, Cambridgeshire, UK). The output of the laser was attenuated to avoid saturating the PSD using a neutral density filter (OD 1.3 NE513B; Thorlabs Cambridgeshire, UK).

The optic cage system which maintains the optic axis of the collimator, lens and neutral density filter was mounted on a system of two automated translation stages (M110.1DG \& M122.2DD; Physik Instrumente, Bedford, UK) which allowed precise readout from each of the cantilevers in the array in a sequential manner. The motion of the stages is controlled by a LabVIEW interface. An additional microtranslation stage (Gothic Arch 9061-XYZ; Newport, Calif, USA) allows initial positioning of the laser spot at the tip of the cantilever prior to the start of an experiment.

The laser beam is deflected from the tip of the cantilever onto a linear position sensitive detector (PSD, Sitek, Partille, Sweden). The current output from the PSD is converted to a voltage with a cutoff frequency of $2 \mathrm{MHz}$ (due to the response time of the optical detector). The output from the PSD is amplified (SR560 Low-Noise Preamplifier; Stanford Research Systems, Calif, USA) then digitised (NI PCI 5112; National Instruments, Tex, USA) before being analysed with the output from the frequency generator in a LabVIEW program where the time domain signal is converted into a frequency spectrum. The peaks of the spectrum correspond to the flexural resonant modes of the cantilever.

The entire device is housed inside a box which is kept at a constant temperature of $23.0 \pm 0.1^{\circ} \mathrm{C}$ to avoid any drifts in the measurement due to temperature changes. The temperature is kept constant by a fuzzy logic controller which is implemented in LabVIEW.

Cantilever arrays were taken at random from the production wafers and multiple measurements of the first four flexural resonance modes were taken for the $7 \mu \mathrm{m}$ thick cantilevers and of the first seven modes of the $2 \mu \mathrm{m}$ thick cantilevers.
The resonance peaks obtained can be described by a simple harmonic oscillator model [22]

$$
A(f)=A_{\mathrm{bl}}+\frac{A_{0} f_{R, n}^{2}}{\sqrt{\left(f^{2}-f_{R, n}^{2}\right)^{2}+f^{2} f_{R, n}^{2} / Q^{2}}},
$$

where $A_{\mathrm{bl}}$ is the amplitude of the baseline, $A_{0}$ is the zero frequency amplitude, $f$ is the frequency, $f_{R, n}$ is the resonance frequency of mode $n$, and $Q$ is the quality factor. The $Q$ factor and resonance frequencies were extracted from the best fit of the resonance peaks with the above model using a LevenbergMarquardt algorithm [23]. The mean and standard deviation of the resonance frequencies and $Q$ factors of each of the modes was then calculated from the fitted data.

\section{Results and Discussion}

3.1. Numerical Calculations. Van Eysden and Sader's extended viscous [18] and compressible fluid models [20] were used to predict the resonance frequency and $Q$ factor of modes of the $7 \mu \mathrm{m}$ thick cantilevers which were below $2 \mathrm{MHz}$ and the modes of the $2 \mu \mathrm{m}$ thick cantilevers below $1 \mathrm{MHz}$. The compressible fluid model is very sensitive to the thickness of the cantilever for a given length. As shown above the thickness of the cantilevers in the array can vary significantly across the production wafer. As a result of the variation of thicknesses observed the models were used to predict the $Q$ factors and resonance frequencies predicted for the middle and the limits of the range of thicknesses $(7.2 \pm$ $0.5 \mu \mathrm{m}$ for the $7 \mu \mathrm{m}$ thick cantilevers and $1.972 \pm 0.005 \mu \mathrm{m}$ for the $2 \mu \mathrm{m}$ thick cantilevers).

The material and fluid properties were chosen to match the experimental conditions. Young's Modulus of Si: $169 \mathrm{GPa}$; density of $\mathrm{Si}\left(\rho_{\mathrm{Si}}\right): 2330 \mathrm{~kg} / \mathrm{m}^{3}$; density of air $\left(\rho_{\text {air }}\right)\left(\right.$ at RT): $1.1839 \mathrm{~kg} / \mathrm{m}^{3}$; viscosity of air (at RT): $1.78 \times$ $10^{-5} \mathrm{~kg} /(\mathrm{m} \mathrm{s})$; speed of sound in air (at RT): $346.18 \mathrm{~m} / \mathrm{s}$. 


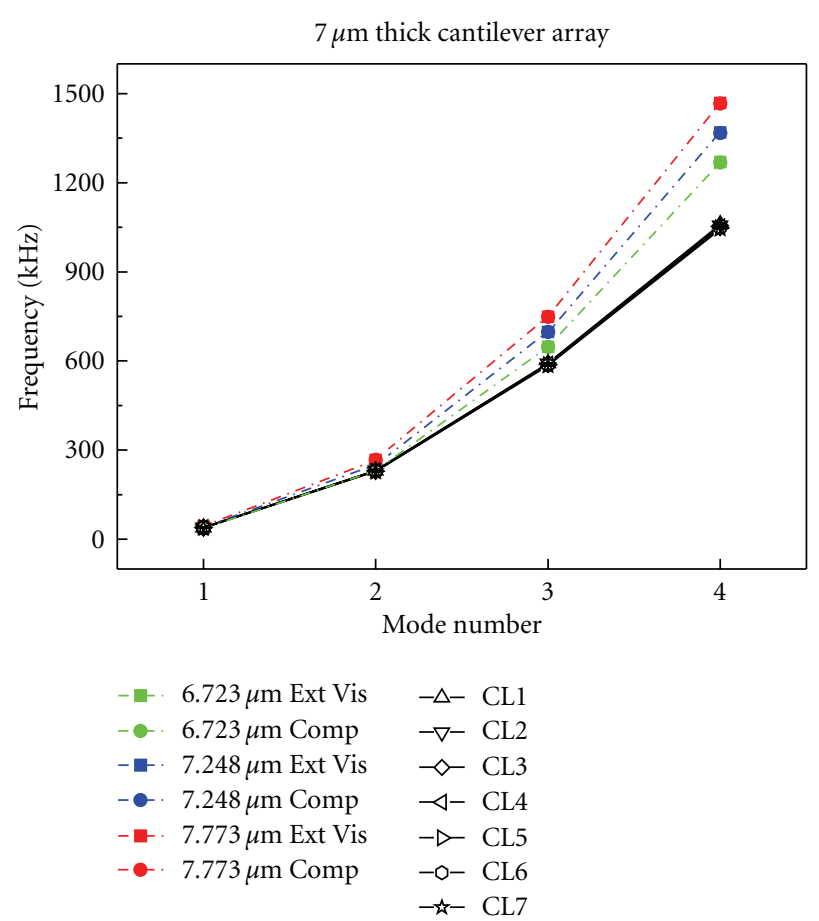

(a)

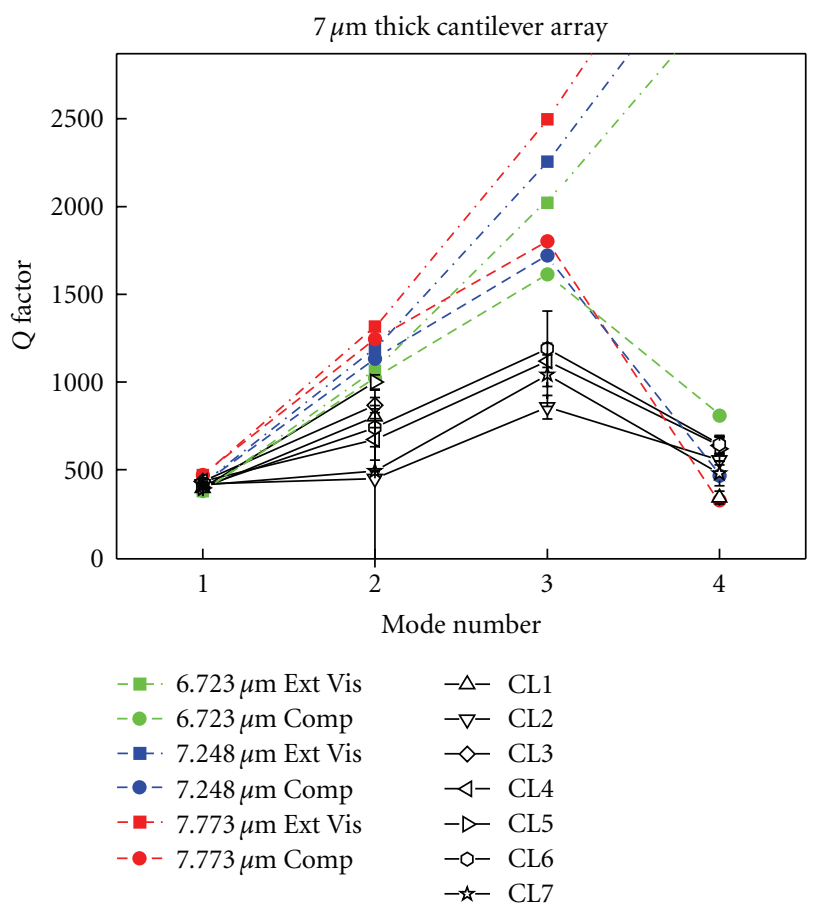

(b)

FIGURE 3: Resonance frequency and $Q$ factor versus mode number comparison between theory and experiment for the $7 \mu \mathrm{m}$ thick cantilevers. The solid square symbols with dotted lines correspond to the extended viscous model, while the solid circles with dashed lines correspond to the compressible fluid model. The open symbols with solid lines correspond to the experimental data. In the frequency plot the experimental data are overlapping.

The general equations for the resonance frequency and $Q$ factor of a given mode are $[18,20]$

$$
\begin{aligned}
f_{R, n} & =\frac{f_{\mathrm{vac}, n}}{\sqrt{1+\left(\pi \rho_{\mathrm{air}} b / 4 \rho_{\mathrm{Si}} t\right) \Gamma_{r}\left(f_{R, n}, n\right)}}, \\
Q & =\frac{\left(4 \rho_{\mathrm{Si}} t / \pi \rho_{\mathrm{air}} b\right)+\Gamma_{r}\left(f_{R, n}, n\right)}{\Gamma_{i}\left(f_{R, n}, n\right)},
\end{aligned}
$$

where $t$ is the thickness of the cantilever, $b$ is the width of the cantilever, $f_{\mathrm{vac}, n}$ is the vacuum resonance frequency of mode $n$ of the cantilever, and $\Gamma\left(f_{R, n}, n\right)$ is the dimensionless hydrodynamic function and the subscripts $r$ and $i$ refer to the real and imaginary components, respectively. The calculations of the $Q$ factor and resonance frequencies required finding the hydrodynamic function for each of the models (it is this term that the compressibility of the fluid affects). This involved solving the systems of linear equations given in [18, equation (11)] and in [20, equation (7)]. The integer $M$ described in the models was chosen to be 36 and was shown to provide sufficient convergence of the solution for the higher modes of vibration (data not shown). For further information on the characteristics of these functions and their convergence see references $[18,20$, 24]. Mathematica 8.0 was used to perform the calculations.

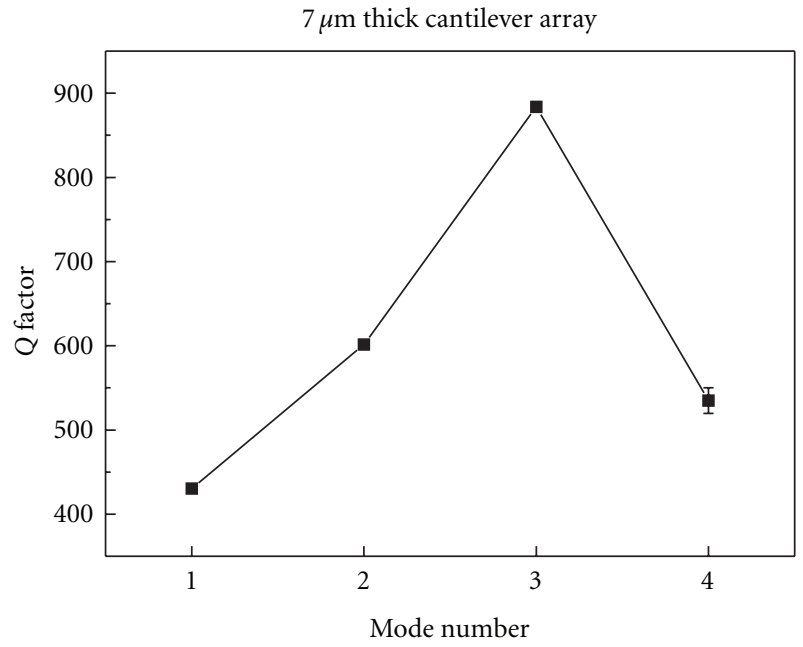

Figure 4: Repeated measurement of the $Q$ factor versus mode number for a single cantilever without removing the array between measurements. The standard deviation of the $Q$ factors for modes $1-3$ is $0.003 \%$ and the standard deviation for mode 4 is $0.02 \%$. This indicated that the previously observed larger standard deviations were due to difference in the coupling between the cantilever and the piezo between experiments.

\subsection{Comparison between Theory and Experiment}

3.2.1. $7 \mu \mathrm{m}$ Thick Cantilevers. It was found that there was a decrease in the $Q$ factor of the seven cantilever beams 


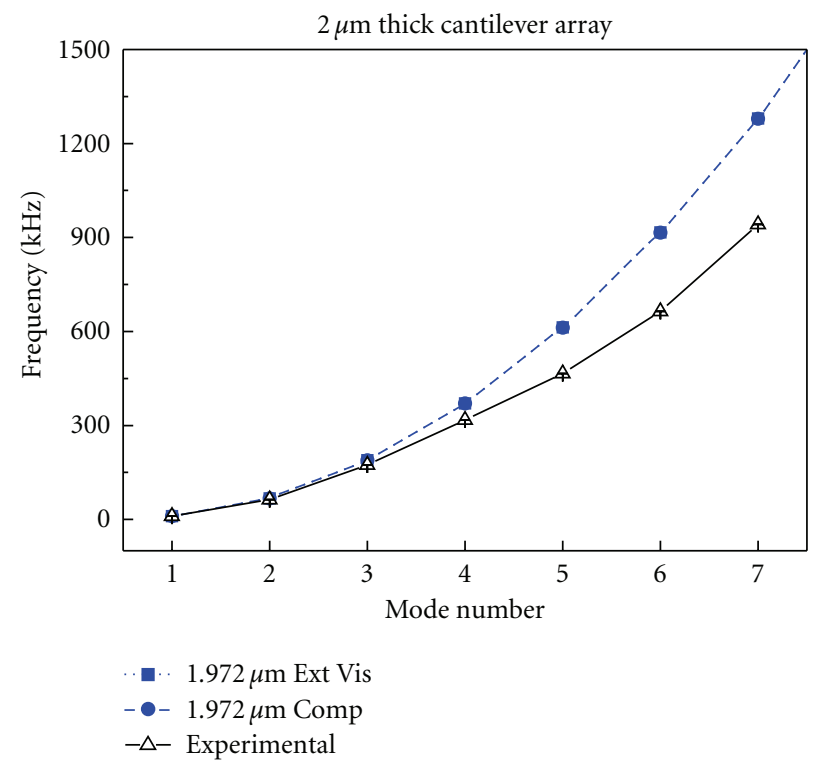

(a)

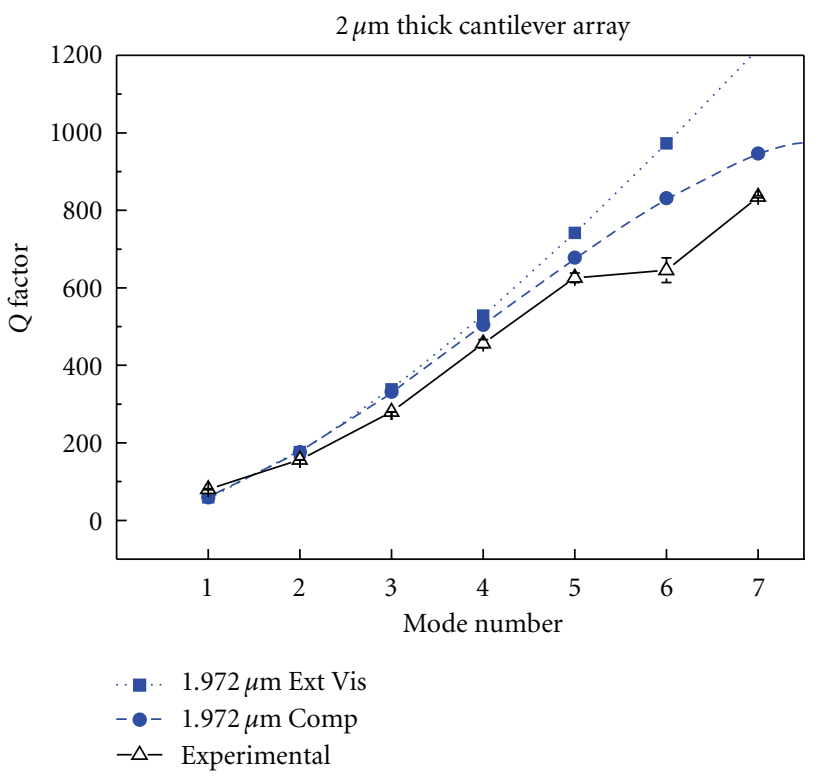

(b)

FIGURE 5: Resonance frequency and $Q$ factor versus mode number comparison between theory and experiment for the $2 \mu \mathrm{m}$ thick cantilever. The solid square symbols with dotted lines correspond to the extended viscous model, while the solid circles with dashed lines correspond to the compressible fluid model. The open symbols with solid lines correspond to the experimental data.

between the third and fourth modes which agrees with the prediction of the scaling analysis mentioned above that the "coincidence point" being the $n=3$ mode. This decrease in $Q$ factor can be clearly seen in Figure 3 .

The large error bars of the experimental data shown in Figure 3 correspond to the standard deviation of the $Q$ factor as measured from five experiments where the cantilever was unclamped and reclamped between experiments and is an indication of the coupling between the piezo and the cantilever. The standard deviation of the resonance frequencies measured for each cantilever were on the order of $0.001 \%$. There was a difference of $\sim 400 \mathrm{~Hz}$ in the fundamental frequency between cantilever 1 and cantilever 7. A similar decrease was also noted in the higher modes of the cantilevers and is an indication that there is a noticeable difference in thickness of the cantilevers within the array.

The resonance frequencies measured experimentally at the first mode agreed well with the models, however as the mode number increased the measured frequencies were increasingly lower than those predicted by the models. The lower frequencies observed are consistent with a cantilever which is longer than the cantilevers used here, but shorter than the cantilever and hinge section added together ( $\sim 620 \mu \mathrm{m}$ total length, data not shown). The strong dependence of the predictions of the compressible fluid model on the thickness of the cantilever can be observed in Figure 3.

A repeat of the experiment where the cantilever was not removed from the holder between measurements is shown in Figure 4. Here ten measurements were taken and it should be noted that the standard deviation is considerably smaller.
3.2.2. $2 \mu \mathrm{m}$ Thick Cantilevers. The "coincidence point" predicted for a $2 \mu \mathrm{m}$ thick cantilever was mode 12 with a resonance frequency of $\sim 3.6 \mathrm{MHz}$. Using the current device it is not possible to observe the flexural resonance modes at such a high frequency, and therefore only the modes up to $1 \mathrm{MHz}$ were observed. The hinge portion of the array is relatively thicker for these arrays than for the $7 \mu \mathrm{m}$ thick cantilever arrays and as such should have less of an effect on the dynamics of the cantilever.

Figure 5 shows the comparison between the experimental data and the predictions of the extended viscous and compressible fluid models. It is clear that the resonance frequencies are still below those predicted by the models; however they match better than for the $7 \mu \mathrm{m}$ thick cantilever array ( $16 \%$ compared to $29 \%$ difference at mode 4 ). This is an indication that the comparatively thinner hinge portion of the array is making a significant contribution to the dynamics of the $7 \mu \mathrm{m}$ thick cantilever arrays.

It is clear from Figure 5 that there are differences in the predicted $Q$ factors of the two models well below the "coincidence point". The experimental values match well with those predicted by the compressible fluid model $(20 \%$ lower for the compressible fluid model compared to $75 \%$ lower for the extended viscous at mode 7).

3.3. Discussion. It is clear that the experimental data agrees qualitatively with the predictions of the compressible fluid model of Van Eysden and Sader, but that absolute quantitative agreement is not demonstrated here. Deviations of the resonance frequency and $Q$ factors of the cantilevers between the predictions from the compressible fluid model 
and the experimental findings could be due to the hinge of the cantilever being only approximately twice or three times the thickness of the cantilever (Figure 1). This may not provide a sufficiently stiff support and there may be some degree of mechanical coupling between the hinge and the cantilever beam. This is significantly more noticeable for the $7 \mu \mathrm{m}$ thick cantilevers where the hinge is comparatively thinner and as such is an indication that the hinge is the cause of the observed deviations from the compressible fluid model predictions. The models are based on an ideal cantilever extending from a fixed support and as such should not be expected to predict exactly the behaviour of cantilevers with a hinge design such as the one used here, however theoretical geometric assumptions are not always translatable into physical microfabricated devices.

Another possible reason for a qualitative and not a quantitative result could be that the model is based on thermal actuation of the cantilever beam and here a piezo actuator is used to amplify the motion of the cantilever, and while efforts are made to keep the cantilever operating within the linear regime of the vibrations this may not be $100 \%$ successful. It should also be noted that the model is valid for cantilevers with a large aspect ratio and here the cantilevers used to conduct the experiment have a ratio of 5 which places them very near the boundary for which the theory is valid.

In conclusion it was observed that there is at least qualitative agreement with the compressible fluid model for practical microcantilevers with a thickness to length ratio of $\sim 7: 500$ and an aspect ratio of 5. The prediction from the scaling analysis of Van Eysden and Sader of a "coincidence point" at mode 3 for the $7 \mu \mathrm{m}$ thick cantilever is accurate and is clearly observed in the experimental data. The lower than predicted $Q$ factors and resonant frequencies are likely attributed to the geometry and design of the hinge portion of the cantilever. The compressible fluid model should be considered when planning experiments involving the use of higher resonant modes of relatively thick microcantilevers in air.

\section{Acknowledgments}

The authors would like to thank John Sader and Anthony Van Eysden for discussions regarding their compressible fluid model. The SEM images were taken in the Advanced Microscopy Laboratory, Trinity Technology and Enterprise Campus, Dublin 2, Ireland under the framework of the INSPIRE program, funded by the Irish Government's Programme for Research in Third Level Institutions, Cycle 4, National Development Plan 2007-2013. This work was supported by Science Foundation Ireland under the CSET scheme SFI08/CE/I1432 and PI scheme SFI/09IN/1B2623.

\section{References}

[1] G. Binnig, C. F. Quate, and C. Gerber, "Atomic force microscope," Physical Review Letters, vol. 56, no. 9, pp. 930-933, 1986.

[2] R. McKendry, J. Zhang, Y. Arntz et al., "Multiple labelfree biodetection and quantitative DNA-binding assays on a nanomechanical cantilever array," Proceedings of the National Academy of Sciences of the United States of America, vol. 99, no. 15, pp. 9783-9788, 2002.

[3] F. Huber, M. Hegner, C. Gerber, H. J. Guntherodt, and H. P. Lang, "Label free analysis of transcription factors using microcantilever arrays," Biosensors \& Bioelectronics, vol. 21, no. 8, pp. 1599-1605, 2006.

[4] L. M. Lechuga, J. Tamayo, M. Alvarez et al., "A highly sensitive microsystem based on nanomechanical biosensors for genomics applications," Sensors and Actuators B, vol. 118, no. 1-2, pp. 2-10, 2006.

[5] J. Mertens, C. Rogero, M. Calleja et al., "Label-free detection of DNA hybridization based on hydration-induced tension in nucleic acid films," Nature Nanotechnology, vol. 3, no. 5, pp. 301-307, 2008.

[6] D. Ramos, M. Arroyo-Hernandez, E. Gil-Santos et al., "Arrays of dual nanomechanical resonators for selective biological detection," Analytical Chemistry, vol. 81, no. 6, pp. 2274-2279, 2009.

[7] P. S. Waggoner, M. Varshney, and H. G. Craighead, "Detection of prostate specific antigen with nanomechanical resonators," Lab on a Chip, vol. 9, no. 21, pp. 3095-3099, 2009.

[8] N. Backmann, C. Zahnd, F. Huber et al., "A label-free immunosensor array using single-chain antibody fragments," Proceedings of the National Academy of Sciences of the United States of America, vol. 102, no. 41, pp. 14587-14592, 2005.

[9] T. Braun, M. K. Ghatkesar, N. Backmann et al., "Quantitative time-resolved measurement of membrane protein-ligand interactions using microcantilever array sensors," Nature Nanotechnology, vol. 4, no. 3, pp. 179-185, 2009.

[10] B. Ilic, Y. Yang, and H. G. Craighead, "Virus detection using nanoelectromechanical devices," Applied Physics Letters, vol. 85, no. 13, pp. 2604-2606, 2004.

[11] N. Nugaeva, K. Y. Gfeller, N. Backmann, H. P. Lang, M. Duggelin, and M. Hegner, "Micromechanical cantilever array sensors for selective fungal immobilization and fast growth detection," Biosensors \& Bioelectronics, vol. 21, no. 6, pp. 849856, 2005.

[12] K. Y. Gfeller, N. Nugaeva, and M. Hegner, "Rapid biosensor for detection of antibiotic-selective growth of Escherichia coli," Applied and Environmental Microbiology, vol. 71, no. 5, pp. 2626-2631, 2005.

[13] K. Y. Gfeller, N. Nugaeva, and M. Hegner, "Micromechanical oscillators as rapid biosensor for the detection of active growth of Escherichia coli," Biosensors \& Bioelectronics, vol. 21, no. 3, pp. 528-533, 2005.

[14] D. Ramos, J. Tamayo, J. Mertens, M. Calleja, L. G. Villanueva, and A. Zaballos, "Detection of bacteria based on the thermomechanical noise of a nanomechanical resonator: origin of the response and detection limits," Nanotechnology, vol. 19, no. 3, Article ID 035503, 2008.

[15] M. K. Ghatkesar, V. Barwich, T. Braun et al., "Higher modes of vibration increase mass sensitivity in nanomechanical microcantilevers," Nanotechnology, vol. 18, no. 44, Article ID 445502, 2007.

[16] F.-J. Elmer and M. Dreier, "Eigenfrequencies of a rectangular atomic force microscope cantilever in a medium," Journal of Applied Physics, vol. 81, no. 12, pp. 7709-7714, 1997.

[17] J. E. Sader, "Frequency response of cantilever beams immersed in viscous fluids with applications to the atomic force microscope," Journal of Applied Physics, vol. 84, no. 1, pp. 6476, 1998. 
[18] C. A. Van Eysden and J. E. Sader, "Frequency response of cantilever beams immersed in viscous fluids with applications to the atomic force microscope: arbitrary mode order," Journal of Applied Physics, vol. 101, no. 4, Article ID 044908, 2007.

[19] M. K. Ghatkesar, T. Braun, V. Barwich et al., "Resonating modes of vibrating microcantilevers in liquid," Applied Physics Letters, vol. 92, no. 4, Article ID 043106, 2008.

[20] C. A. Van Eysden and J. E. Sader, "Frequency response of cantilever beams immersed in compressible fluids with applications to the atomic force microscope," Journal of Applied Physics, vol. 106, no. 9, Article ID 094904, 2009.

[21] C. A. V. Van Eysden and J. E. Sader, "Compressible viscous flows generated by oscillating flexible cylinders," Physics of Fluids, vol. 21, no. 1, Article ID 013104, 2009.

[22] J. W. M. Chon, P. Mulvaney, and J. E. Sader, "Experimental validation of theoretical models for the frequency response of atomic force microscope cantilever beams immersed in fluids," Journal of Applied Physics, vol. 87, no. 8, pp. 3978-3988, 2000.

[23] K. Levenberg, "A method for the solution of certain problems in least squares," Quarterly of Applied Mathematics, vol. 2, pp. 164-168, 1944.

[24] C. A. Van Eysden and J. E. Sader, "Resonant frequencies of a rectangular cantilever beam immersed in a fluid," Journal of Applied Physics, vol. 100, no. 11, Article ID 114916, 2006. 

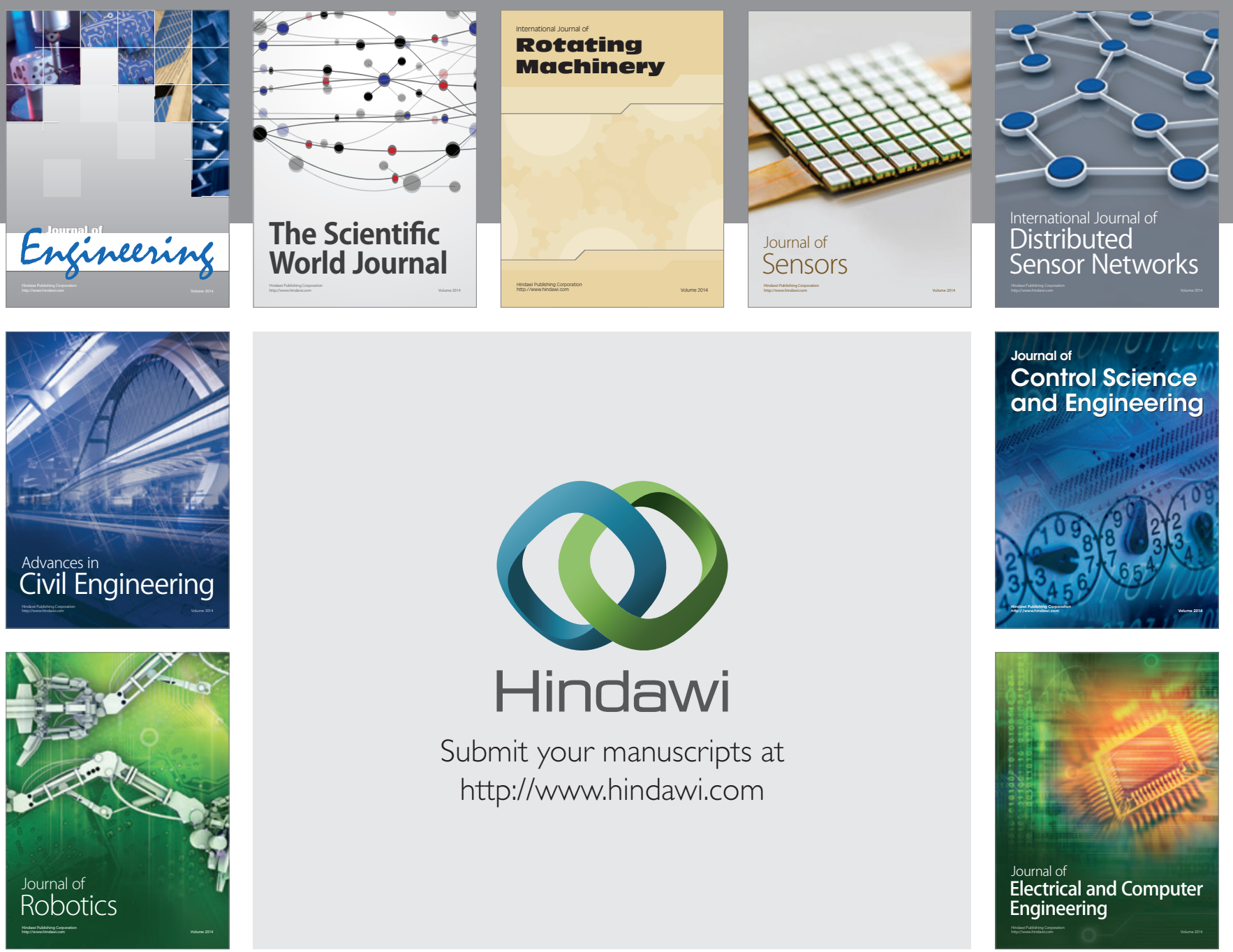

Submit your manuscripts at

http://www.hindawi.com
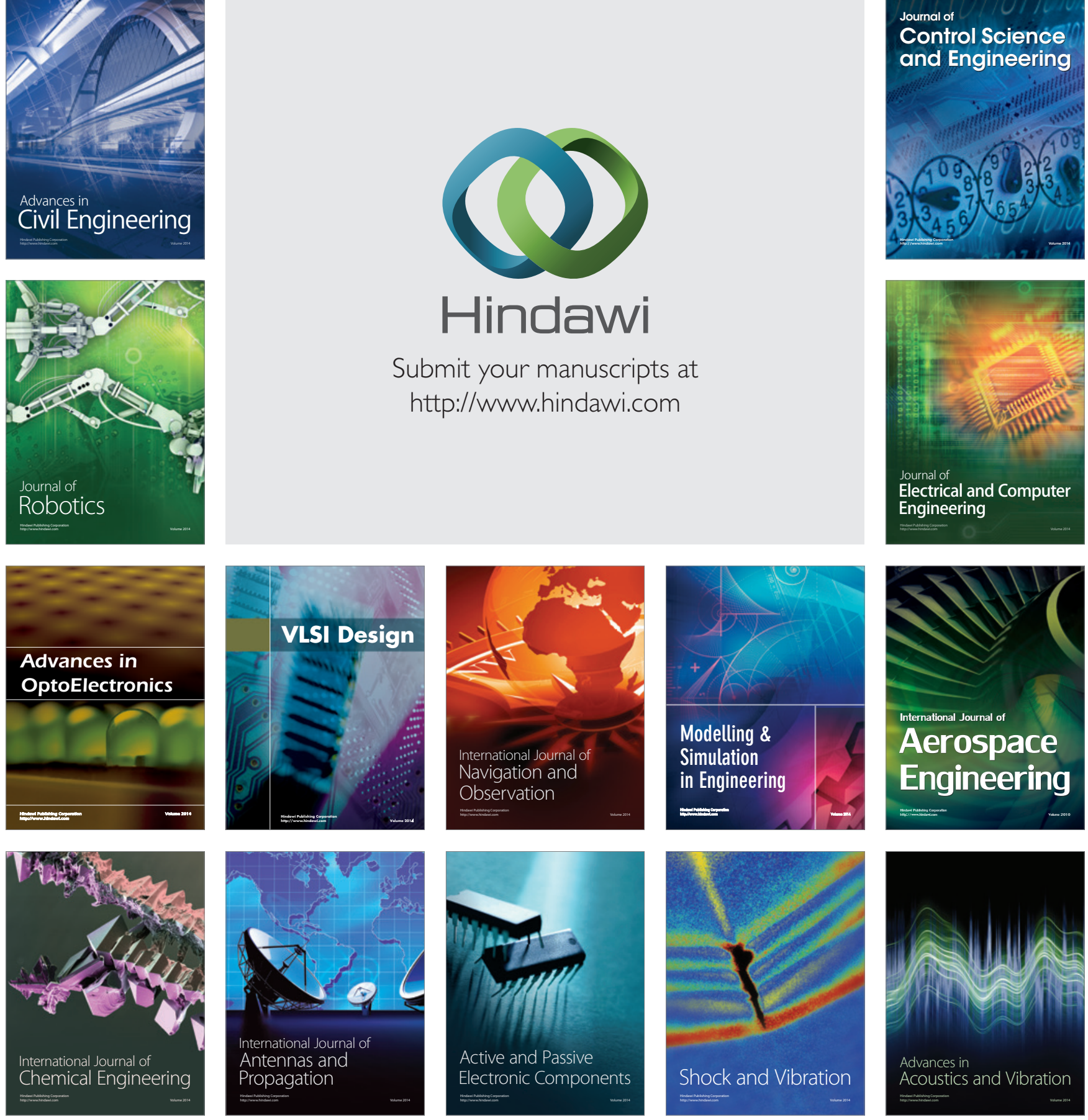